\title{
UNIQUE FACTORIZATION FOR POLYNOMIALS
}

\author{
CHARLES H. GIFFEN
}

Suppose $R$ is a unique factorization domain and $R[x]$ is not. Let $p$ be a polynomial in $R[x]$ minimal in degree with respect to the property that $p$ has two essentially different prime (irreducible polynomial) factorizations. We may assume $p=f_{1} f_{2} \cdots f_{r}=g_{1} g_{2} \cdots g_{s}$, where all $f_{i}$ and $g_{j}$ are primes with $f_{i} \neq u g_{j}$ for any unit $u$ and where

$$
\begin{gathered}
m=\operatorname{deg} f_{1} \geqq \operatorname{deg} f_{2} \geqq \cdots \geqq \operatorname{deg} f_{r}, \\
n=\operatorname{deg} g_{1} \geqq \operatorname{deg} g_{2} \geqq \cdots \geqq \operatorname{deg} g_{8} .
\end{gathered}
$$

and $n \geqq m>0$. Call $a, b$ the coefficients of $x^{m}, x^{n}$ in $f_{1}, g_{1}$ respectively, and let

$$
q=a p-b f_{1} x^{n-m} g_{2} \cdots g_{s}
$$

On one hand

$$
\begin{aligned}
q & =a f_{1} f_{2} \cdots f_{r}-b f_{1} x^{n-m} g_{2} \cdots g_{s} \\
& =f_{1}\left(a f_{2} \cdots f_{r}-b x^{n-m} g_{2} \cdots g_{s}\right),
\end{aligned}
$$

while on the other hand

$$
\begin{aligned}
q & =a g_{1} g_{2} \cdots g_{s}-b f_{1} x^{n-m} g_{2} \cdots g_{s} \\
& =\left(a g_{1}-b f_{1} x^{n-m}\right) g_{2} \cdots g_{s} .
\end{aligned}
$$

From this we see that if $q=0$, then $a g_{1}=b f_{1} x^{n-m}$. If, however, $q \neq 0$, then note $\operatorname{deg}\left(a g_{1}-b f_{1} x^{n-m}\right)<\operatorname{deg} g_{1}$ and hence $\operatorname{deg} q<\operatorname{deg} p$, so that $q$ must have a unique factorization into primes, some of which are $g_{2}, \cdots, g_{s}$ and $f_{1}$. But then $f_{1}$ must be a factor of $\left(a g_{1}-b f_{1} x^{n-m}\right)$ and hence also of $a g_{1}$. Therefore, in either case, $a g_{1}=f_{1} h$ for some polynomial $h$, so that, since $f_{1}$ is prime and $\operatorname{deg} f_{1}>0$ and $\operatorname{deg} a=0$, then $a$ is a factor of $h$; hence, $h=a h^{\prime}$ for some polynomial $h^{\prime}$, and $a g_{1}=f_{1} a h^{\prime}$, or $g_{1}=f_{1} h^{\prime}$. This shows that $f_{1}$ is a factor of $g_{1}$, which is impossible by our assumptions. Hence, $R[x]$ must indeed be a unique factorization domain. This generalization to polynomials of a well-known direct proof for unique factorization in the natural numbers arose out of an incidental remark by Professor C. W. Curtis in one of his courses at the University of Wisconsin.

Princeton University and

UNIVERSITY OF WISCONSIN

Received by the editors February 7, 1962 and, in revised form, January 7, 1963. 